NOTA CIENTÍFICA

\title{
Succinea peruviana (Gastropoda) en la dieta de la lagartija de las Lomas Microlophus tigris (Sauria) en la Reserva Nacional de Lachay, Lima, Perú
}

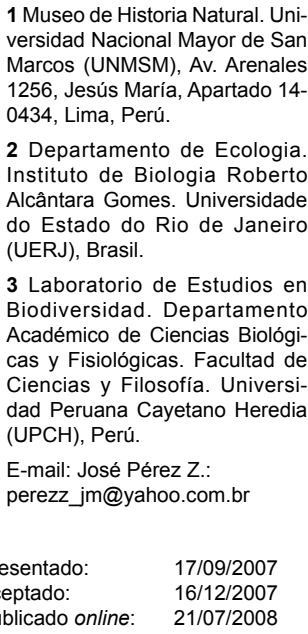

1 Museo de Historia Natural. Universidad Nacional Mayor de San arcos (UNMSM), Av. Arenales 1256, Jesús María, Apartado 14

Departamento de Ecologia. Instituto de Biologia Roberto Alcântara Gomes. Universidade do Estado do Rio de Janeiro (UERJ), Brasil.

3 Laboratorio de Estudios en Académico de Cienciartamento cas y Fisiológicas. Facultad de Ciencias y Filosofía. Universidad Peruana Cayetano Heredia (UPCH), Perú.

E-mail: José Pérez Z

Presentado: $\quad 17 / 09 / 2007$

Aceptado: $\quad 16 / 12 / 2007$

Las lagartijas consumen frecuentemente caracoles, incluso existen algunas especializadas en el consumo de estos moluscos, como por ejemplo Dracaena guianensis en Amazonia y especies del género Cyclodomorphus en Australia (Pianka y Vitt, 2003). Sin embargo, en el Desierto Costero Peruano hasta la fecha no existen informes de alguna especie de lagartija que consuma caracoles.

Las lagartijas del Desierto Costero Peruano tienen una dieta caracterizada principalmente por el consumo de artrópodos (Dixon y Huey, 1970; Dixon y Wrigth, 1975; Péfaur y LópezTejeda, 1983; Pérez y Jhancke, 1998; Pérez, 2005a; Pérez y Balta, 2007). Sin embargo, el patrón generalista de sus dietas posibilita el consumo de una amplia diversidad de itemes alimenticios, que en algunos casos incluyen plantas (Dixon y Wrigth, 1975; Pérez, 2005a, Pérez y Balta, 2007) y vertebrados (Pérez, 2005b; Pérez y Balta, 2005a; Pérez y Balta, 2005b).

La lagartija de las Lomas Microlophus tigris Tschudi, 1845 es endémica del Desierto Costero Peruano (Dixon y Wrigth, 1975; Carrillo e Icochea, 1995), y es el reptil más frecuente en la Reserva Nacional de Lachay (RNL) (Pérez, 2005a), así como en las Lomas de la costa central peruana (Dixon y Wrigth, 1975). El caracol Succinea peruviana es la única especie del género conocida para la costa central del Perú, y está presente en los ecosistemas de Lomas como la RNL (Aguilar, 1985; Ramírez, 1992; Ramírez et al., 1999).

En el presente trabajo damos a conocer el consumo del caracol $S$. peruviana por la lagartija de las Lomas $M$. tigris en el mesohábitat de Lomas con árboles en la RNL.

Se realizaron colectas de la lagartija de las Lomas $M$. tigris en el mesohábitat de Lomas con árboles en la RNL (11 ${ }^{\circ} 20^{\prime} \mathrm{S}, 77^{\circ} 19^{\prime}$ W) durante los meses de enero-marzo del 2004, época seca de Lomas (Torres y López, 1981). Esta colecta fue realizada como parte de una investigación sobre la ecología de $M$. tigris y del geko de las Lomas Phyllodactylus lepydopigus (Pérez, 2005a).

El contenido estomacal de las lagartijas colectadas fue preservado en alcohol $70 \%$. El contenido estomacal fue analizado con la ayuda de un microscopio estereoscópico. La identificación de la especie de caracol fue posible debido a que los individuos consumidos no estaban totalmente destruidos, y aunque no fue posible observar la concha, la parte blanda mantenía la forma típica de Succinea.

Se analizaron 33 estómagos de $M$. tigris, en tres estómagos (9\%), se registraron restos de 16 individuos del caracol S. peruviana. Las lagartijas que consumieron caracoles fueron individuos adultos, dos hembras y un macho (MUSM 24566 , $\mathrm{SVL}=59,9 \mathrm{~mm}, \mathrm{LT}=60,2 \mathrm{~mm}, \mathrm{~W}=7,1 \mathrm{~g} ;$ MUSM 24567 中,

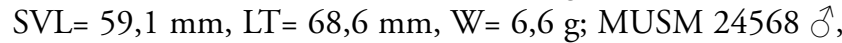
$\mathrm{SVL}=67,1 \mathrm{~mm}, \mathrm{LT}=96,9 \mathrm{~mm}, \mathrm{~W}=10,5 \mathrm{~g})$. Colectamos estas tres lagartijas en zonas con vegetación arbustiva y arbórea. Las lagartijas colectadas se encuentran depositadas en la colección del Departamento de Herpetología del Museo de Historia Natural de la Universidad Nacional Mayor de San Marcos, y el material malacológico recuperado se encuentra depositado en el Departamento de Malacología y Carcinología del mismo museo.

En la RNL el caracol $S$. peruviana se distribuye entre los 400 - 600 m de altitud, y durante la época seca de Lomas estiva principalmente en la parte baja de arbustos y árboles, pudiendo quedar cubierto por una delgada capa de materia orgánica (Ramírez et al., 1999). El período de estivación de $S$. peruviana coincidió con la época de colecta de las lagartijas evaluadas. En la RNL han sido registrados diez especies de caracoles (Aguilar, 1976; Ramírez et al., 1999), de los cuales $S$. peruviana seria la única especie de caracol que podría ser consumida en esta época por $M$. tigris, debido a su localización en la base de las plantas, es decir, al alcance de estas lagartijas.

Bostryx conspersus es la especie de caracol más frecuente en la RNL (Ramírez et al., 1999) y es encontrada estivando en la misma época que $S$. peruviana. Sin embargo $M$. tigris consumió a $S$. peruviana, ya que $B$. conspersus estiva generalmente enterrada, dificultando su captura. Adicionalmente a esto, la mayor resistencia de la concha de B. conspersus, comparada con $S$. peruviana, también dificultaría su consumo. 
Por lo tanto, se deduce que $M$. tigris debe haber realizado una búsqueda activa para encontrar a Succinea peruviana, removiendo la materia orgánica (restos de vegetación) que cubren a esta especie de caracol.

\section{Agradecimientos}

Agradecemos a Juan Carlos Jordán y Emilio Fuentes por la ayuda en el trabajo de campo, a la Jefatura y todo el personal de Reserva Nacional de Lachay (INRENA), por la colaboración durante el trabajo en campo. JPZ contó con el apoyo financiero parcial de la Fundaçáo de Amparo a Pesquisa do Estado do Rio de Janeiro (FAPERJ) mediante la Beca de Postgrado, $\mathrm{N}^{\circ}$ Processo : E-26/151.388/2003.

\section{Literatura citada}

Aguilar, P. 1985. Fauna de las Lomas costeras del Perú. Boletín de Lima 7(41): 17-28.

Carrillo, N. y J. Icochea. 1995. Lista taxonómica preliminar de los reptiles vivientes del Perú. Publicaciones del Museo de Historia Natural de la Universidad Nacional Mayor de San Marcos Serie A: Zoología 49: 1-27.

Dixon, J. \& R. Huey. 1970. Systematics of the lizards of the Gekkonid genus Phyllodactylus of mainland South America. Contribution in Science The Natural History Museum of Los Angeles 192: 1-78.

Dixon, J. \& J. Wrigth. 1975. A review of the lizards of the iguanid genus Tropidurus in Peru. Contribution in Science The Natural History Museum of Los Angeles 271: 1-39.

Péfaur, J. \& E. López-Tejeda. 1983. Ecological notes on the lizard Tropidurus peruvianus in southern Peru. Journal of Arid Environments 6: 155-160.
Pérez Z., J. \& K. Balta. 2007. Ecología de la comunidad de saurios diurnos de la Reserva Nacional de Paracas, Ica, Perú. Rev. peru. biol. número especial 13(3): 169 - 176.

Pérez Z., J. 2005a. Ecología de duas espécies de lagartos simpátricos em uma formação vegetal de Lomas no Deserto Costeiro Peruano Central. Dissertação (Grau de Mestre) - Instituto de Biologia Roberto Alcântara Gomes, Universidade do Estado de Rio de Janeiro. Rio de Janeiro, Brasil. 78 p.

Pérez Z., J. 2005b. Microlophus peruvianus. Cannibalism. Herpetological Review 36(1): 63.

Pérez Z., J. y K. Balta. 2005a. Microlophus theresiae. Saurophagy. Herpetological Review 36(1): 63.

Pérez Z., J. y K. Balta. 2005b. Microlophus quadrivittatus. Cannibalism. Herpetological Review 36(2): 180.

Pérez Z., J. y J. Jahncke. 1998. Saurios como consumidores de ectoparásitos de aves guaneras. Boletín del Instituto del Mar del Perú 17(1-2): 81-86.

Pianka, E. \& L. Vitt.2003. Lizards Windows to the Evolution Diversity. University of California Press. 346 pp.

Ramírez, R. 1992. Esporocisto de Leucochloridium (Trematoda, Leucochloridiidae) en Succinea peruviana (Mollusca, Succineidae). Anales Inst. Biol. Univ. Nac. Autón. México, Ser. Zool. 63(2): 173-177.

Ramírez, R., K. Caro, S. Córdova, J. Duárez, A. Cano, C. Arana y J. Roque.1999. Respuesta de Bostryx conspersus y Succinea peruviana (Mollusca:Gastropoda) al evento "El Niño 1997-1998” en las Lomas de Lachay (Lima, Perú). Revista Peruana de Biología, Lima. Volumen Extraordinario (El Niño 1997-98 y su Impacto sobre los Ecosistemas Marino y Terrestre): 143-151.

Torres, J. y C. López. 1981. Productividad primaria en las lomas de la costa central del Perú. Boletín de Lima 3(14): 54-63. 